\title{
Diversity Antenna Design for Compact Devices of Iot Uses
}

\author{
P.Viswanath ${ }^{1}$, J. Hema Latha ${ }^{2 *}$, M. Likitha ${ }^{2}$, K. Bhargavi ${ }^{2}$, Ch. Saranya ${ }^{2}$, J. Shalem Raj ${ }^{2}$ \\ ${ }^{I} M$-Tech, Assistant Professor \\ ${ }^{1,2}$ St. Ann's College of Engineering \& Technology, Chirala.
}

*Corresponding Author: J. Hema Latha, St. Ann's College of Engineering \& Technology, Chirala.

\begin{abstract}
Now-a-days, Internet of Things (IOT) plays a major role in the wireless communications which redefines the scope of the wireless communication. As the concept of compact devices, which are capable of sensing and relaying data, are in great needs. This work presents a dual antenna configuration for the different IOT uses which are used in the widespread of compact platforms. Diversity antennas are adopted to mitigate multipath fading situations in indoor and on-body propagation environments. Diversity antenna that uses two or more antennas to improve the quality and reliability of wireless communication which is one of the diversity scheme. Two open-ended slots are arranged on a $30 \times 30 \mathrm{~mm}^{2}$ system board to minimize the device profile. Near the slot's openings matching is accomplished with the coupled feed. Two slots are arranged in a perpendicular configuration of reduced coupling. By the simulation and measurements results we can obtain the satisfied matching conditions. The coupling level is little high because the two antennas are placed on ground of a limited sizes. This dual antenna configuration makes the system more accurate and lessening the coupling.
\end{abstract}

Keywords: Diversity Antenna, MIMO (Multiple-Input \& Multiple-Output), IOT (Internet of Things), IOT Devices (Bluetooth, WLAN.etc,) Dual Antenna Configuration, ISM band.

\section{INTRODUCTION}

Wireless Communication is the process of sending information from one point to another, the communication system which uses the present wide variety of electrical and electronics shows a significant impact on the modern society. .

As the technology is growing day-by-day the impact on wireless technology also increasing. The wireless body area networks contains sensors attached to clothing or even implanted under the skin. The numerous innovative applications to improve the health care as well as the quality of life can be offered by the wide variety usage of sensors and the connections capability of the wireless streams. An IOT device is a wireless connecting device to a established network inorder to transmit data. As the versatile increase in the IOT devices in the present world, due to this impact so many companies had identified this IOT technology and implementing the miniaturized and integrated communication systems and modules. The IOT devices usage had increased in any stream for the easy enabling and data transmission purpose. IOT devices are part of a scenario in which every device inter relate with the every other device in an environment to the automatic world and communicate more and more usable data to users, particular systems for the data projections etc..

The dual antenna configuration system is well followed in the present technology because the usage of dual antenna increases the performance of the system and the transmission of data is more reliable. The MIMO technology is effective in the multipath mitigating situations and then it is implementing in the recent technologies such as different modules.

This project proposes a MIMO antenna, which comprises of two miniaturized slot antennas works under the ISM band for the IOT devices with the projected frequency of $(2.4 \sim 3 \mathrm{GHz})$, works for the Bluetooth, Wi-Fi, WLAN. It employs for the network gadgets such as WLAN and Bluetooth protocols for the data transmission. 


\section{ANTENNA DESIGN}

The proposed design is shown in fig.1. A quarter wavelength slot is placed $2 \mathrm{~mm}$ away from the board edge of a $0.8 \mathrm{~mm}$ thick FR4 substrate. The FR4 board dimensions are $30 \mathrm{~mm}$ x $30 \mathrm{~mm}$, it emulates the system board of a wearable device. Simulations were conducted with the CST software, which is a package for designing, simulating and optimizing electromagnetic systems. It is a full-wave numerical EM tool based on the finite element method.

The slot contains a metal strip of length L2 in the middle. For the proposed antenna design the slot and metal strip are at the broad edge of the substrate. Important geometric parameters of the slot and the metal strip are marked on the figure 1 as shown .L1 is the slot length. L2 is the metal strip length. The slot has a width of $1.5 \mathrm{~mm}$ from substrate and the internal metal strip has the width of $0.5 \mathrm{~mm}$. The difficulty is moving the slot to the board edge is the diminished bandwidth and the degraded matching condition. It is reduced by decreasing slot length and diminishing the feed position in to the slot. The slots and the metal strip are placed in the edge of the board because in the middle we can fabricate or place the IC or sensing modules which are used for the operation. In the on-body network applications the back side of the design has the direct contact with the body in the case of wearable devices. Due to this the radiation occurs on the body, in-order to maintain this radiation efficiency performance a slot is placed on the back side of the antenna design at the top position.

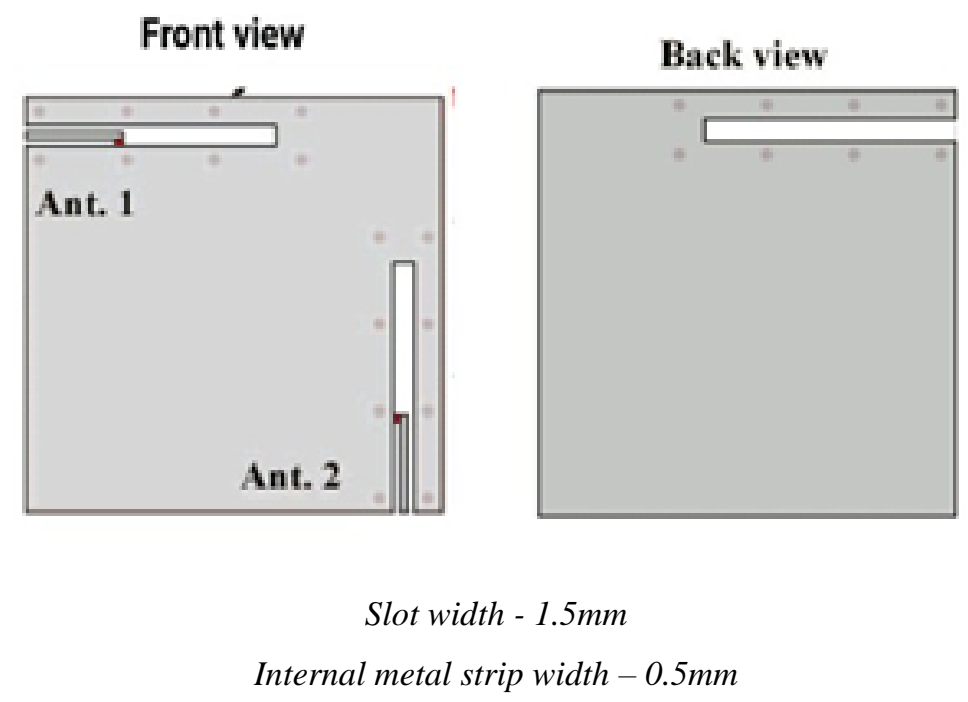

Fig1. Geometry of the proposed antenna design in the front and back view.

In the proposed design the slot length $\mathrm{L} 1$ is varied by changing the length from $17 \mathrm{~mm}-19 \mathrm{~mm}$. The strip length is adjusted and by varying this the resonant frequency is also changed, it will vary from 2.45 to $2.3 \mathrm{GHz}$. The internal metal strip length $\mathrm{L} 2$ is also varied and the value varies from $11 \mathrm{~mm}-$ $7 \mathrm{~mm}$. Then the resonant frequency is increased to some extent than the original frequency obtained.

Here the proposed design is performed in the different cases. In the first case the antennas are placed in edges of the board in the horizontal and vertical as shown in the figure above. And in the other cases the antennas are placed in the horizontal position in one side of board in one case and in another case antennas are placed in horizontal and vertical position on the same side as shown in the figure.

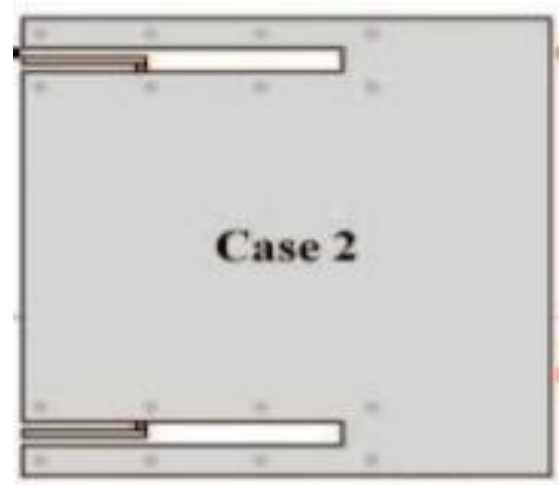




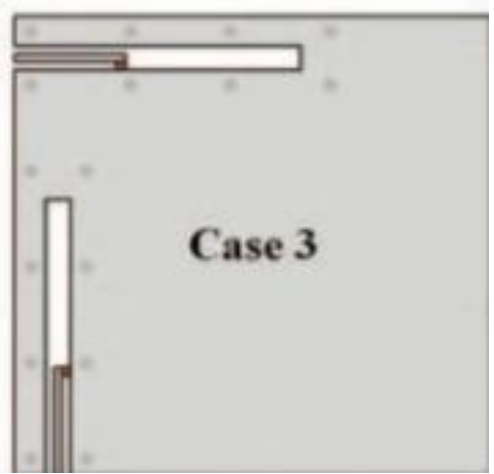

By performing this different cases we can obtain the different type of simulation results.

\section{Simulation Results}

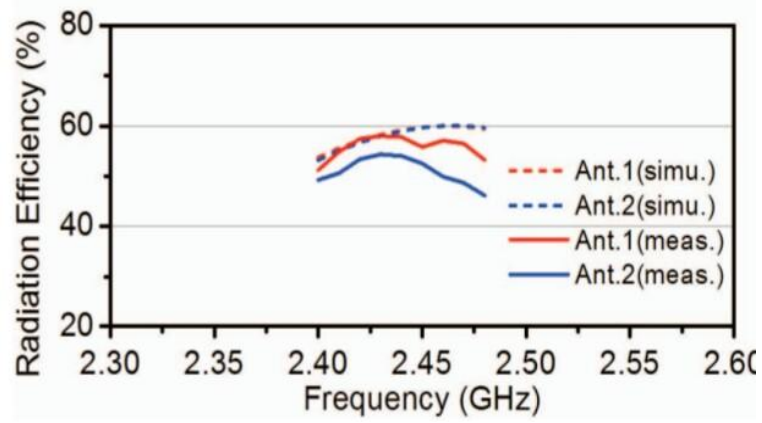

Fig2. Radiation efficiency of the two antenna systems.

The radiation efficiency of the two antenna system is observed in the above figure which are between the $50-60 \%$ in the $2.4 \sim 2.48 \mathrm{GHz}$.

\section{Radiation Patterns of two Antenna Configuration}
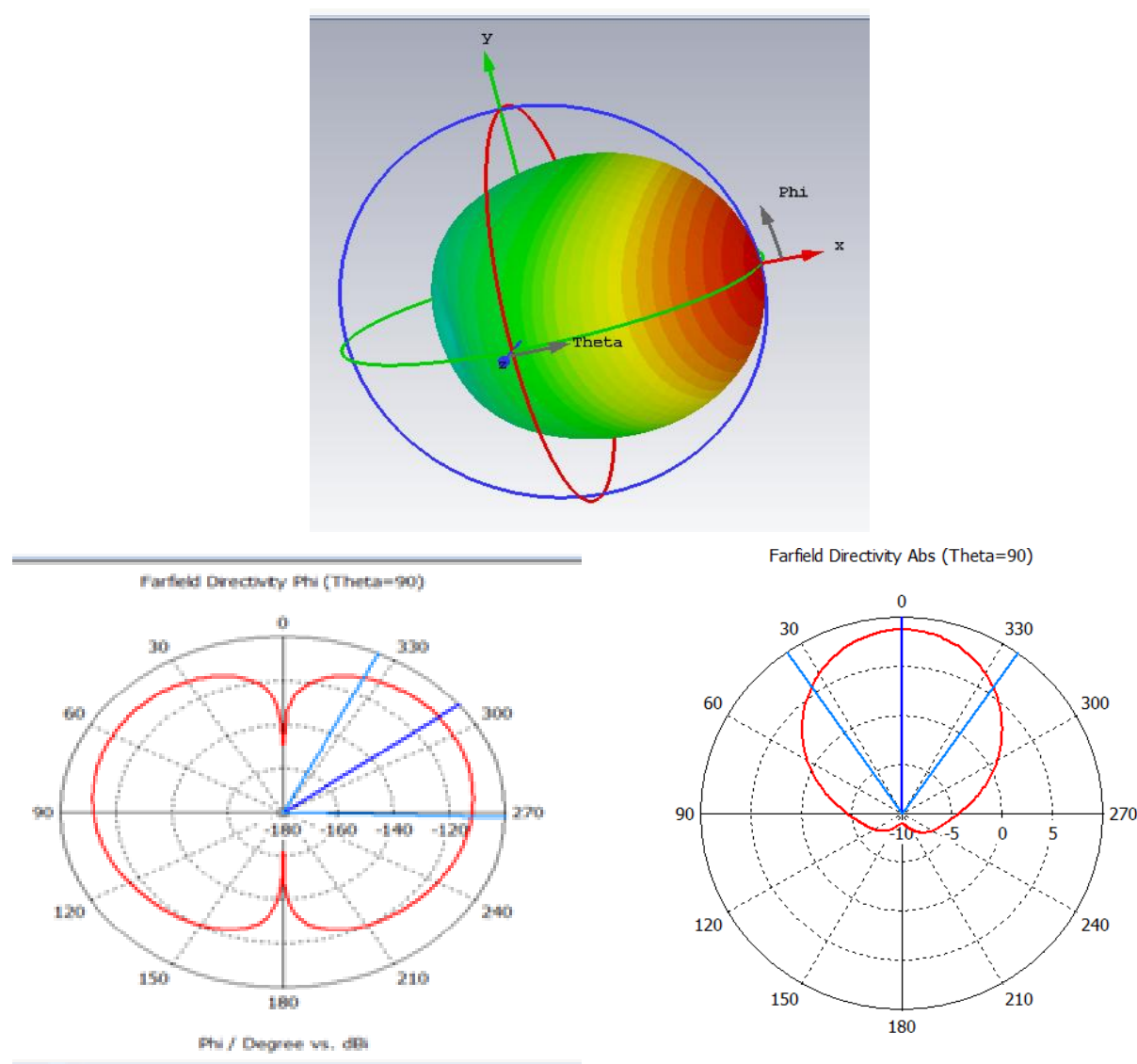

Phi / Denree ve dRi 


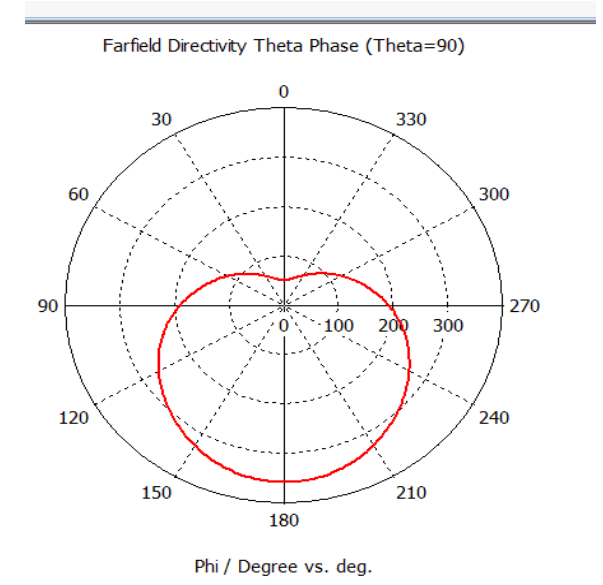

\section{S-Parameters}
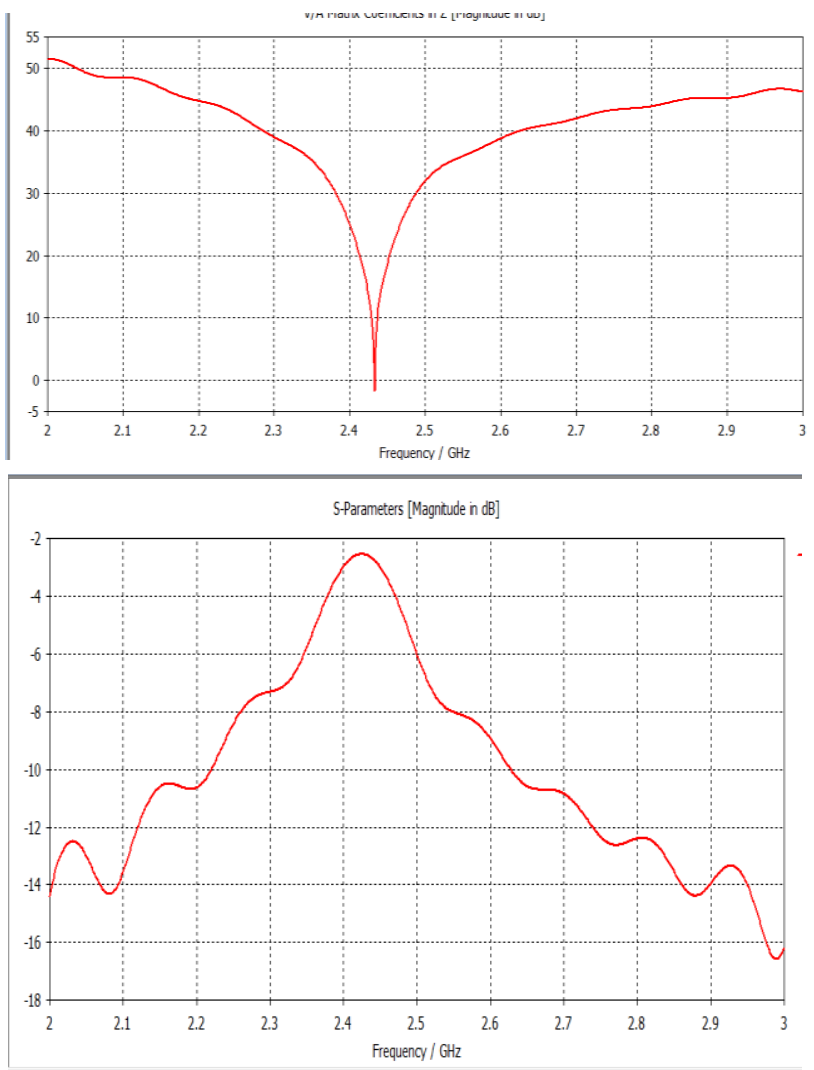

The above two figures determines the s-parameters of the proposed antenna for dual antenna configuration. Here the obtained $-12 \mathrm{~dB}$ is enough to cover the frequency of $2.4 \sim 2.48 \mathrm{GHz}$. Which is used for the Bluetooth and WLAN applications.

\section{VSWR:}

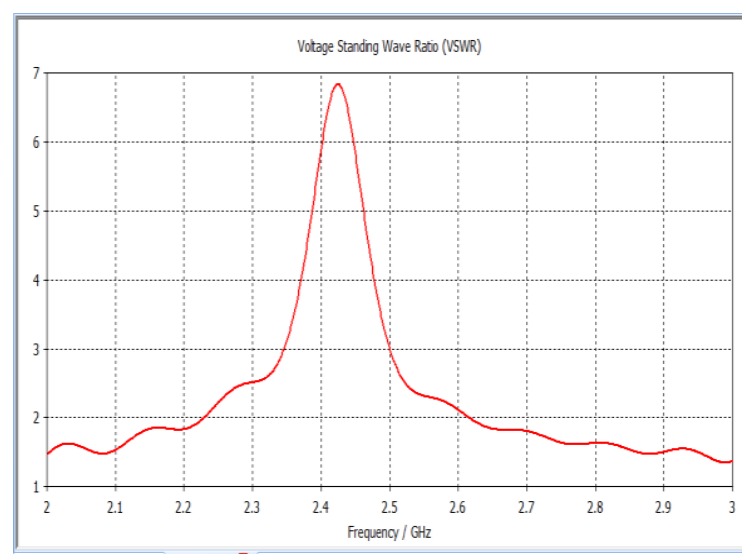


The above figure determines the voltage standing wave ratio of the two antenna configuration of the design.

\section{CONCLUSION}

This paper proposes a MIMO antenna system which composes of a two miniaturized slot antennas that are placed on a $30 \mathrm{~mm} \times 30 \mathrm{~mm}$ board dimensions. The radiation efficiency of the antenna greater than the 50\% in-order to improve the performance in the fading situations. It is used in the range of Bluetooth and WLAN networks in the way to development of iot devices.

\section{REFERENCES}

[1] Z. J. Jin, T. Y. Yun, "Compact Wideband Open-End Slot Antenna with Inherent Matching", IEEE Antennas Wireless Propag. Lett., vol. 13, 2014.

[2] S. Dumanli, C. J. Railton, D. L. Paul, "A slot antenna array with low mutual coupling for use on small mobile terminals", IEEE Trans. Ant. Propag., vol. 59, no. 5, pp. 1512-1520, May. 2011.

[3] M. Sonkki, E. Antonino-Daviu, M. Ferrando- Bataller, E. T. Salonen, "Planar wideband polarization diversity antenna for mobile terminals", IEEE Antennas Wireless Propag. Lett., vol. 10, pp. 939-942, 2011.

[4] M. Sonkki, E. Antonino-Daviu, M. Cabedo-Fabrés, M. Ferrando-Bataller, E. T. Salonen, "Improved planar wideband antenna element and its usage in a mobile MIMO system", IEEE Antennas Wireless Propag. Lett., vol. 11, pp. 826-829, 2012.

[5] M. Karaboikis, C. Soras, G. Tsachtsiris, and V. Makios, "Compact dual-printed inverted-F antenna diversity systems for portable wireless devices," IEEE Antennas Wireless Propag. Lett., vol. 3, pp. 9-14, 2004.

[6] T. Ohishi, N. Oodachi, S. Sekine, and H. Shoki, "A method to improve the correlation and the mutual coupling for diversity antenna," presented at the IEEE Antennas and Propagation Society Int. Symp., Washington, DC, Jul. 2005.

[7] S. Dossche, S. Blanch, and J. Romeu, "Optimum antenna matching to minimise signal correlation on a two-port antenna diversity system,"IET Electron. Lett., vol. X. Wang, Z. Feng, and K. M. Luk, "Pattern and polarization diversity.

[8] Antenna with high isolation for portable wireless devices," IEEE Antennas Wireless Propag. Lett., vol. 8, pp. 209-211, 2009.40, no. 19, pp. 1164-1165, Sep. 2004.

[9] L. Zheng and D. N. C. Tse, "Diversity and multiplexing: A fundamental tradeoff in multiple-antenna channels,” IEEE Trans. Inf. Theory, vol.49, no. 5, pp. 1073-1096, May 2003.

[10] R. G. Vaughan and J. B. Andersen, "Antenna diversity in mobile communications," IEEE Trans. Veh. Technol., vol. VT-36, no. 4, pp.149-172, Nov. 1987.

[11] C.B. Dietrich, K.Dietze, J. R. Nealy, and W. L. Stutzman, "Spatial, polarization, and pattern diversity for wireless handheld terminals," IEEE Trans. Antennas Propag., vol. 49, no. 9, pp. 1271-1281, Sep. 2001

[12] X.Wang, Z. Feng, and K. M. Luk, "Pattern and polarization diversity antenna with high isolation for portable wireless devices," IEEE Antennas Wireless Propag. Lett., vol. 8, pp. 209-211, 2009.

[13] F. Ferrero, A. Diallo, C. Luxey, and B. Derat, "Pattern diversity versus polarization diversity in UMTS mobile phones," in Proc. 3rd EuCAP, Berlin, Germany, Mar. 23-27, 2009, pp. 1522-1525.

\section{AUTHORS' BIOGRAPHY}

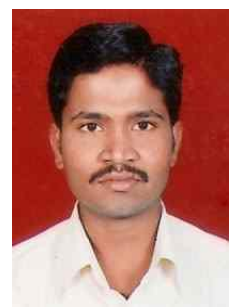

P. Viswanath, M.Tech., Assistant Professor, St. Ann's college of engineering \& technology, Chirala.

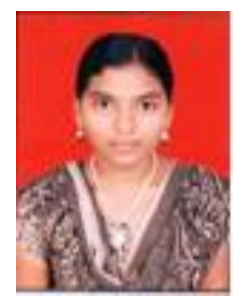

J. Hema Latha, student, Studying final year in the stream of ECE (Electronics \& Communication Engineering) in St. Ann's college of engineering \&Technology, Chirala. 


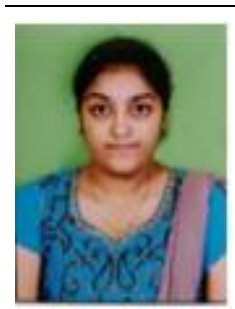

M. Likitha, student, Studying final year in the stream of ECE (Electronics \& Communication Engineering) in St. Ann's college of engineering \&Technology, Chirala.

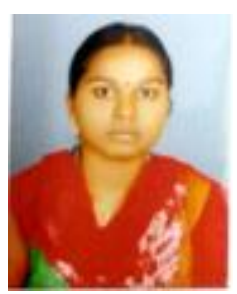

K. Bhargavi, student, Studying final year in the stream of ECE (Electronics \& Communication Engineering) in St. Ann's college of engineering \&Technology, Chirala.

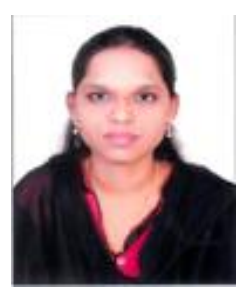

Ch. Saranya, student, Studying final year in the stream of ECE (Electronics \& Communication Engineering) in St. Ann's college of engineering \&Technology, Chirala.

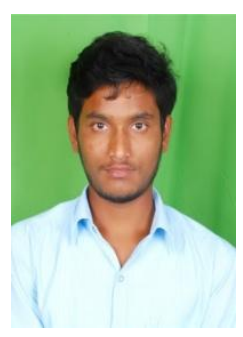

J. Shalem Raju, student, Studying final year in the stream of ECE (Electronics \& Communication Engineering) in St. Ann's college of engineering \&Technology, Chirala.

Citation: Dr. P. Viswanath, et.al. (2018) "Diversity Antenna Design for Compact Devices of Iot Uses", International Journal of Innovative Research in Electronics and Communications (IJIREC), 5(1), pp 12-17. DOI: http://dx.doi. org/10.20431/2349-4050.0501003

Copyright: (C) 2018 Dr. P. Viswanath, This is an open-access article distributed under the terms of the Creative Commons Attribution License, which permits unrestricted use, distribution, and reproduction in any medium, provided the original author and source are credited. 